Fluid Dynamics Research

June 2014, Volume 46 Issue 3 Pages 1417

http://dx.doi.org/10.1088/0169-5983/46/3/031417

http://archimer.ifremer.fr/doc/00202/31360/

(c) 2014 IOP Publishing

\title{
Instability of a two-step Rankine vortex in a reduced gravity QG model
}

\author{
Perrot Xavier ${ }^{1}$, Carton Xavier ${ }^{2,3}$ \\ ${ }^{1}$ Ecole Normale Super, Meteorol Dynam Lab, F-75005 Paris, France. \\ ${ }^{2}$ Univ Bretagne Occidentale, Lab Phys Oceans, F-29200 Brest, France.
}

* Corresponding authors : Xavier Perrot, email address : xperrot@Imd.ens.fr ; Xavier Carton, email address : xcarton@univ-brest.fr

\begin{abstract}
:
We investigate the stability of a steplike Rankine vortex in a one-active-layer, reduced gravity, quasigeostrophic model. After calculating the linear stability with a normal mode analysis, the singular modes are determined as a function of the vortex shape to investigate short-time stability. Finally we determine the position of the critical layer and show its influence when it lies inside the vortex.
\end{abstract}

Keywords : Vortex dynamics, Instability, Normal/Singular modes, Critical layer 


\section{Introduction}

The stability of vortices has long since been studied, with analytical and numerical approaches, in 2D or stratified flows, for stepwise (Rankine) or continuous vorticity distributions, in QG or shallow water models. In this paper we will focus on the stability of a two-step Rankine vortex in a QG model. Different methods can be used to determine vortex stability (normal or singular modes in the linear dynamics, minimization of quadratic functionals in linear or nonlinear dynamics, initial value problems in nonlinear numerical simulations). A usual criterion for the growth of unstable perturbations is that their energy increases. The Rayleigh-Kuo and Charney-Stern criteria for linear instability in 2D or QG flows are based on that constraint; these criteria state that the mean potential vorticity gradient must change sign in/or between layers for instability to develop (Rayleigh 1880, Charney and Stern 1962). The direct computation of the growth rates of normal modes has been performed for a variety of flows in 2D or multilayer fluids (Pedlosky 1985, Sokolovskiy 1997, Carton and Corréard 1999, Carton et al 2010a, b). In this case, the unstable regions, when they exist, are obtained for each mode in the parameter plane.

The instability of a multistep Rankine vortex with normal mode perturbations has been discussed at length in the past years (Stern 1987, Flierl 1988, Morel and Carton 1994). The main result is that the nonlinear evolution of barotropic unstable vortices leads to the ejection of dipoles or to the formation of stable multipoles (for low perturbation modes). We shall now consider a one-and-a-half layer model to study this vortex stability to different perturbations. Firstly we will perform a normal mode analysis. Following this study, we will investigate explosive instability in short-time due to singular modes (Fisher 1998, Rivière et al 2001). Finally we will determine the

position of the critical layers to study the spiraling of vorticity contours for a linearly stable vortex (Balmforth et al 2001).

\section{The stationary solution and the perturbed case}

We consider a two-step Rankine vortex in a one-and-a-half layer incompressible flow in polar representation. Its generic profile is:

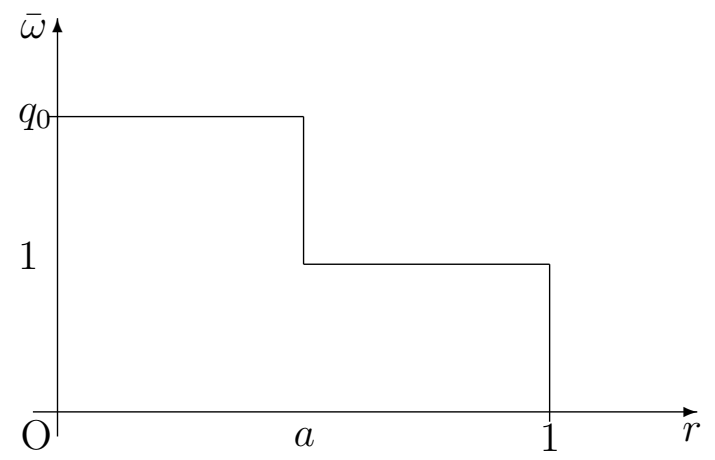

Here $\bar{\omega}=\Delta \bar{\Psi}-\gamma^{2} \Psi$ where $\bar{\omega}$ is the mean potential vorticity, $\bar{\Psi}$ is the mean 
streamfunction and $1 / \gamma=R d=\sqrt{g^{\prime} H} / f_{0}$ is the radius of deformation (with $g^{\prime}$ the reduced gravity, $H$ the height of the layer and $f_{0}$ the coriolis parameter).

This vortex can be decomposed in two parts:
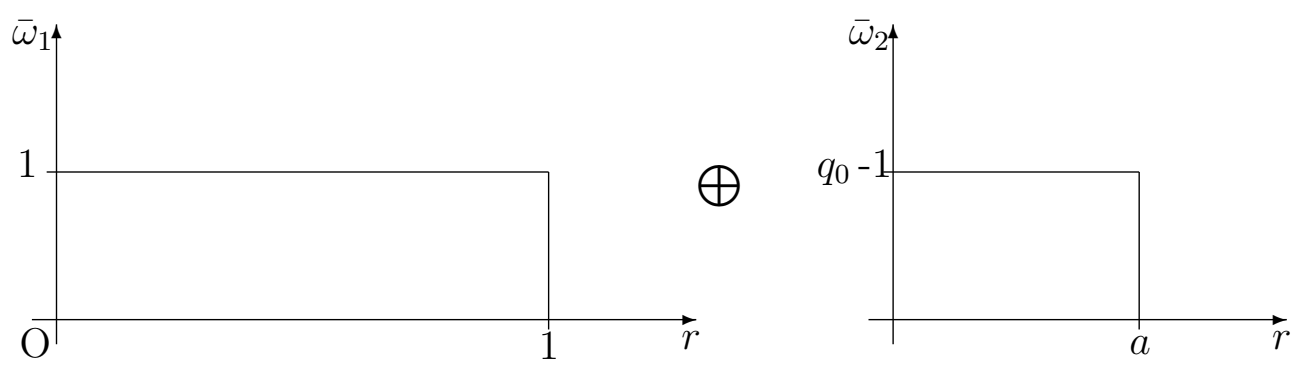

where

$$
\bar{\omega}=\bar{\omega}_{1}+\bar{\omega}_{2},
$$

with

$$
\bar{\omega}_{1}=\Delta \bar{\Psi}_{1}-\gamma^{2} \bar{\Psi}_{1} \quad \text { and } \quad \bar{\omega}_{2}=\Delta \bar{\Psi}_{2}-\gamma^{2} \bar{\Psi}_{2} .
$$

Because of the linearity of the Laplacian operator we obtain:

$$
\bar{\Psi}=\bar{\Psi}_{1}+\bar{\Psi}_{2}
$$

Then we can calculate the mean streamfunction $(\bar{\Psi})$ and the mean velocity $(\bar{U})$ which has just the orthoradial component because of the circular symmetry. We use the continuity of $\bar{\Psi}$ and $\bar{U}_{\theta}$ at the PV fronts. We obtain:

$$
\begin{cases}\bar{\Psi}(r)=\frac{-q_{0}}{\gamma^{2}}+\frac{\left(q_{0}-1\right) a K_{1}(\gamma a)+K_{1}(\gamma)}{\gamma} I_{0}(\gamma r) & r \leq a, \\ \bar{\Psi}(r)=-\frac{1}{\gamma^{2}}\left[1-\gamma K_{1}(\gamma) I_{0}(\gamma r)\right]-\frac{q_{0}-1}{\gamma} a I_{1}(\gamma a) K_{0}(\gamma r) & a \leq r \leq 1, \\ \bar{\Psi}(r)=-\frac{\left(q_{0}-1\right) a I_{1}(\gamma a)+I_{1}(\gamma)}{\gamma} K_{0}(\gamma r) & r \geq 1,\end{cases}
$$

and

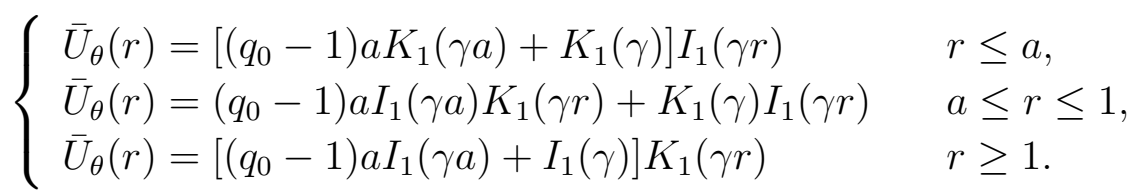

We add a perturbation at the two boundaries of the Rankine vortex:

$$
\begin{aligned}
& \eta_{a}(\theta, t)=\eta_{a 0} e^{i l \theta} A(t), \\
& \eta_{1}(\theta, t)=\eta_{10} e^{i l \theta} A(t),
\end{aligned}
$$

with $\eta_{a}$ and $\eta_{1} \ll 1$.

Then the stream function is:

$$
\Psi=\bar{\Psi}+\psi^{\prime}
$$

where $\psi^{\prime}(r, \theta, t)=\phi(r) e^{i l \theta} A(t)$. The potential vorticity becomes:

$$
\omega(r)=\left(q_{0}-1\right)\left[1-H\left(r-a-\eta_{a}\right)\right]+1-H\left(r-1-\eta_{1}\right),
$$

where $H$ is the Heaviside function. 
We obtain at first order:

$$
\omega^{\prime}=\omega-\bar{\omega}=\left(1-q_{0}\right) \delta\left(r-a-\eta_{a}\right)-\delta\left(r-1-\eta_{1}\right),
$$

where $\delta$ is the Dirac distribution.

$\omega^{\prime}$ vanishes everywhere except at the vortex boundaries. Thus we have $\Delta \psi^{\prime}-\gamma^{2} \psi^{\prime}=0$ nearly everywhere, which implies:

$$
r \frac{\partial}{\partial r}\left(r \frac{\partial \phi(r)}{\partial r}\right)-\left(\gamma^{2} r^{2}+l^{2}\right) \phi(r)=0
$$

The solution of (1) is:

$$
\phi(r)=A I_{l}(\gamma r)+B K_{l}(\gamma r) .
$$

Because $\phi$ is finite at $r=0$ and at $r=+\infty$, we obtain for the three regions of the plane:

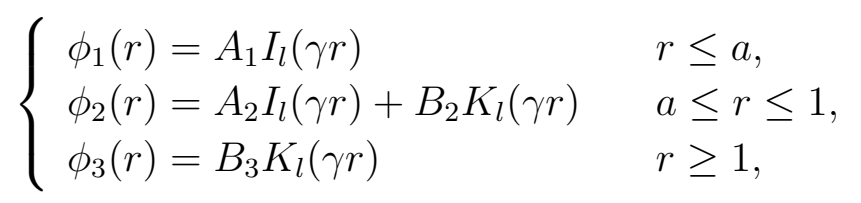

and continuity implies:

$$
\left\{\begin{array}{l}
A_{1}=A_{2}+B_{2} \frac{K_{l}(\gamma a)}{I_{l}(\gamma a)}, \\
B_{3}=A_{2} \frac{I_{l}(\gamma)}{K_{l}(\gamma)}+B_{2}
\end{array}\right.
$$

On the other hand, continuity of $U_{\theta}$ (at $r=a$ and $r=1$ ) implies, at first order, the continuity of $U_{\theta}^{\prime}+\eta \frac{d \bar{U}_{\theta}}{d r}$. We also have $\bar{\omega}=\frac{d \bar{U}_{\theta}}{d r}+\frac{\bar{U}_{\theta}}{r}$ with $\frac{\overline{U_{\theta}}}{r}$ continuous. That implies:

$$
\left[U_{\theta}^{\prime}\right]_{i n t}^{e x t}+\eta\left[\frac{d \bar{U}_{\theta}}{d r}\right]_{i n t}^{e x t}=\left[\frac{d \psi^{\prime}}{d r}\right]_{i n t}^{e x t}+\eta[\bar{\omega}]_{i n t}^{e x t}=0
$$

We deduce from (2) at $r=a$ and $r=1$ two other relations between $A_{1}, A_{2}, B_{2}$ and $B_{3}$ which yield:

$$
\begin{aligned}
& A_{2}=-K_{l}(\gamma) \eta_{10} \quad \text { and } \quad B_{2}=-\left(q_{0}-1\right) a I_{l}(\gamma a) \eta_{a 0} \\
& A_{1}=A_{2}+B_{2} \frac{K_{l}(\gamma a)}{I_{l}(\gamma a)} \quad \text { and } \quad B_{3}=A_{2} \frac{I_{l}(\gamma)}{K_{l}(\gamma)}+B_{2}
\end{aligned}
$$

Finally because $d_{t} \eta=\partial_{t} \eta+\bar{U}_{\theta} \partial_{\theta} \eta=U_{r}^{\prime}$ we obtain the evolution equation of $\left(\eta_{a}, \eta_{1}\right)$ :

$$
\frac{\partial}{\partial t}\left(\begin{array}{l}
\eta_{a} \\
\eta_{1}
\end{array}\right)=i\left[\begin{array}{ll}
A_{11} & A_{12} \\
A_{21} & A_{22}
\end{array}\right]\left(\begin{array}{l}
\eta_{a} \\
\eta_{1}
\end{array}\right),
$$

where the four components of matrix $A$ are:

$$
\begin{aligned}
& A_{11}=l\left[\left(q_{0}-1\right) I_{l}(\gamma a) K_{l}(\gamma a)-\left[\left(q_{0}-1\right) K_{1}(\gamma a)+\frac{K_{1}(\gamma)}{a}\right] I_{1}(\gamma a)\right] \\
& A_{12}=\frac{l}{a} K_{l}(\gamma) I_{l}(\gamma a) \\
& A_{21}=l\left(q_{0}-1\right) a K_{l}(\gamma) I_{l}(\gamma a) \\
& A_{22}=l\left[I_{l}(\gamma) K_{l}(\gamma)-\left[\left(q_{0}-1\right) a I_{1}(\gamma a)+I_{1}(\gamma)\right] K_{1}(\gamma)\right]
\end{aligned}
$$

Equation (3) is the central point of our stability analysis, both for normal and singular modes. 

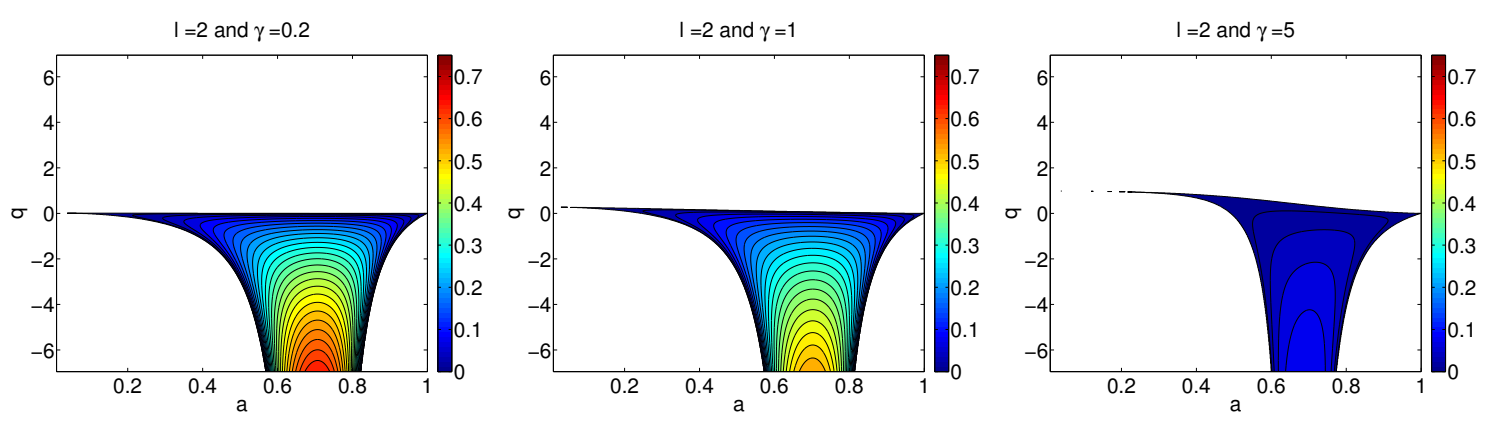

(a) $l=2$
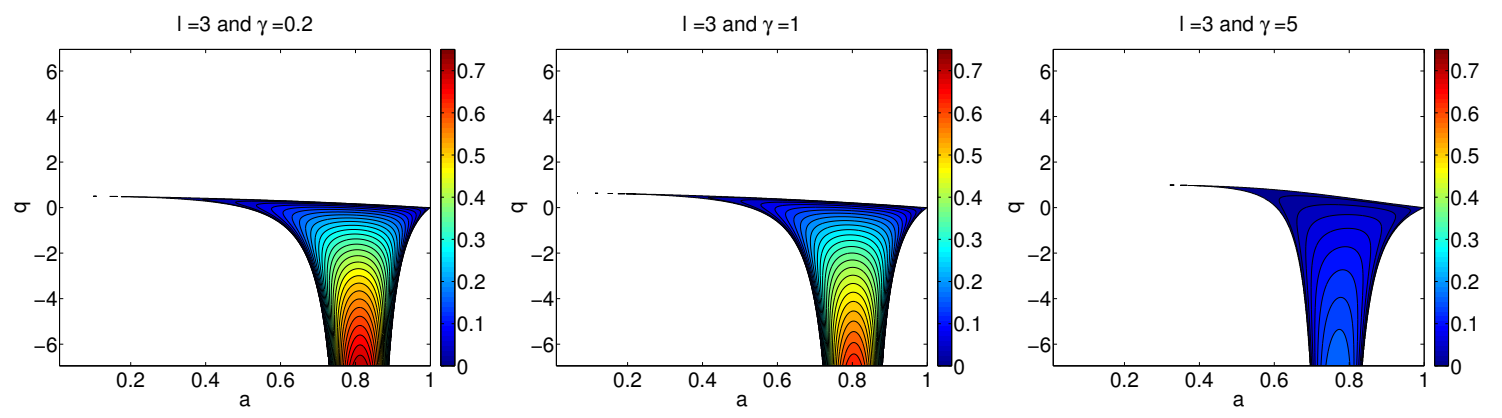

(b) $l=3$
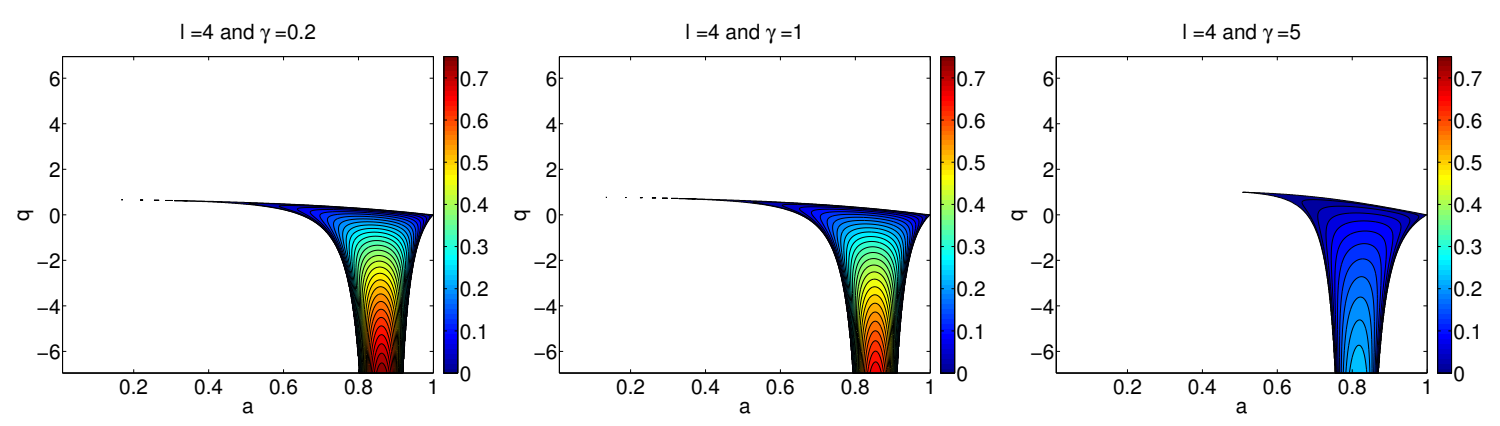

(c) $l=4$

Figure 1. Growth rates of the normal modes in the $\left(a, q_{0}\right)$ plane as a function of $\gamma=0.2,1,5$ and $l=2,3,4$ (white areas correspond to strictly zero growth rate).

\section{The normal modes}

Normal modes grow (or decay) exponentially with time according to $\partial_{t} \eta=\sigma \eta$. Thus, we have $\sigma^{2}-i\left(A_{11}+A_{22}\right) \sigma-A_{11} A_{22}+A_{12} A_{21}=0$, or

$$
\sigma_{ \pm}=\frac{i}{2}\left(A_{11}+A_{22}\right) \pm \frac{i}{2} \sqrt{\Delta}, \quad \Delta=\left(A_{11}-A_{22}\right)^{2}+4 A_{12} A_{21},
$$

which leads to the discriminant for instability

$$
\begin{gathered}
\Delta=l^{2}\left[\left(q_{0}-1\right) I_{l}(\gamma a) K_{l}(\gamma a)-I_{l}(\gamma) K_{l}(\gamma)+\left[\left(q_{0}-1\right) a I_{1}(\gamma a)+I_{1}(\gamma)\right] K_{1}(\gamma)-\right. \\
\left.\quad\left[\left(q_{0}-1\right) K_{1}(\gamma a)+\frac{K_{1}(\gamma)}{a}\right] I_{1}(\gamma a)\right]^{2}+4 l^{2}\left(q_{0}-1\right) K_{l}^{2}(\gamma) I_{l}^{2}(\gamma a),
\end{gathered}
$$


where $q_{0} \in \mathbb{R}, \gamma \in \mathbb{R}^{+}, a \in[0,1]$ and $l \in \mathbb{N}$. Normal mode instability will occur when $\Delta<0$.

It can be easily shown (see below) that these two-step vortices are stable with respect to $l=0$ and $l=1$ normal modes (a similar remark was made for vortices with continuous profiles, by Gent and McWilliams (1986)). Figures 1(a), 1(b), 1(c) show the growth rates in the $\left(a, q_{0}\right)$ plane as a function of $\gamma$, for $l=2,3,4$. As it is well known, higher modes are unstable to stronger shears, induced by narrower external annuli (see for instance Flierl (1988)).

One can also note that, according to the Rayleigh-Kuo criterion, such vortices are stable to normal modes if $q_{0} \geq 1$. For $l=2$, figure 1(a) shows that contrary to the pure barotropic case, the vortex can be unstable for $1 \geq q_{0} \geq 0$. We also notice the development of a horn in the upper left part of the unstable area which grows for larger $\gamma$ and $l$. As shown by Flierl (1988) when $\gamma$ increases the unstable area decreases and approaches to $a=1$ and the values of the growth rates in this area decreases rapidly. Several analytical solutions are now provided.

Specific solutions are :

- $l=0 \Delta=0$ so that $\sigma_{ \pm}=(0,0)$ and the flow is then stable to normal modes.

- $l=1 \Delta=\left[\left(q_{0}-1\right) a+\frac{1}{a}\right]^{2} K_{1}^{2}(\gamma) I_{1}^{2}(\gamma a)$ and $\sigma_{ \pm}=\left(0, i\left[\left(q_{0}-1\right) a+\frac{1}{a}\right] K_{1}(\gamma) I_{1}(\gamma a)\right)$; again the flow is stable to normal mode perturbations.

- $q_{0} \geq 1 \Delta=\left(A_{11}-A_{22}\right)^{2}+4 l^{2}\left(q_{0}-1\right) K_{l}^{2}(\gamma) I_{l}^{2}(\gamma a)$ is positive and the two-step vortices are stable to normal modes.

- $\gamma \gg 1$ All elements of matrix A tend to 0 ; then we have $\Delta \rightarrow 0$ and $\sigma_{ \pm} \rightarrow 0$. This implies a decrease of the growth rates for negative values of $\Delta$.

\section{Singular modes}

Calling $M(t)$ the resolvant of the problem $\partial_{t} \eta=A \eta$, i.e. giving the solution $\eta(t)=$ $M(t) \eta(0)$, singular modes and growth rates are the eigenvectors and eigenvalues of $M^{*} M$ (see Rivière et al (2001)), where $M^{*}$ is the adjoint matrix of $M$. Since $A$ is time-independent and is diagonalized as $A=P \Lambda P^{-1}, M$ is easily obtained as

$$
M=P e^{\Lambda t} P^{-1} .
$$

And we defined:

$$
P=\left[\begin{array}{cc}
1 & 1 \\
a_{+} & a_{-}
\end{array}\right],
$$

with $a_{ \pm}=-\left[i \sigma_{ \pm}+A_{11}\right] / A_{12}$, and

$$
\Lambda=\left[\begin{array}{cc}
\sigma_{+} & 0 \\
0 & \sigma_{-}
\end{array}\right] .
$$



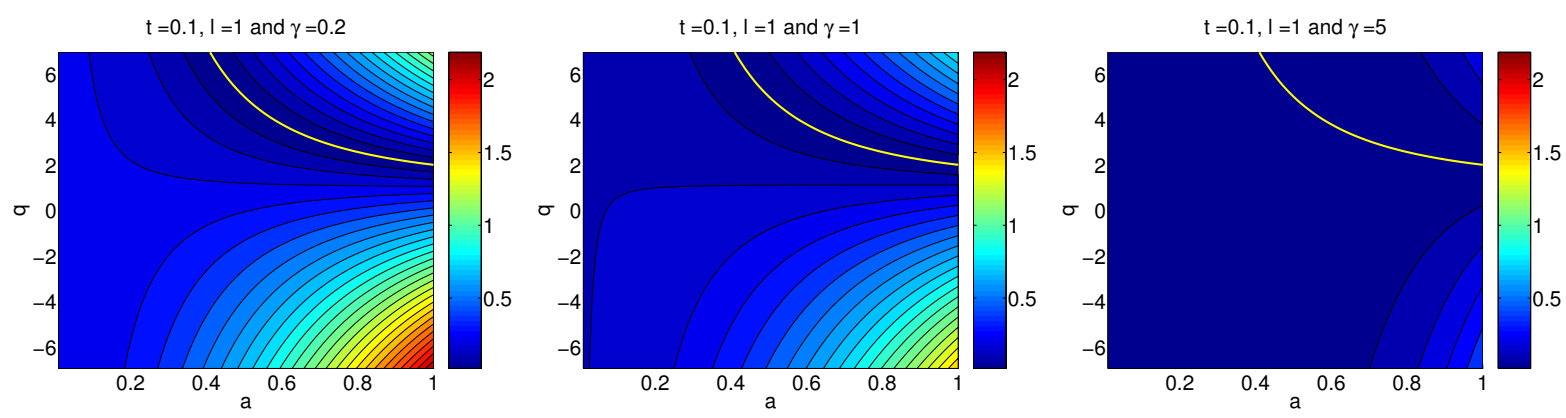

(a) $l=1$
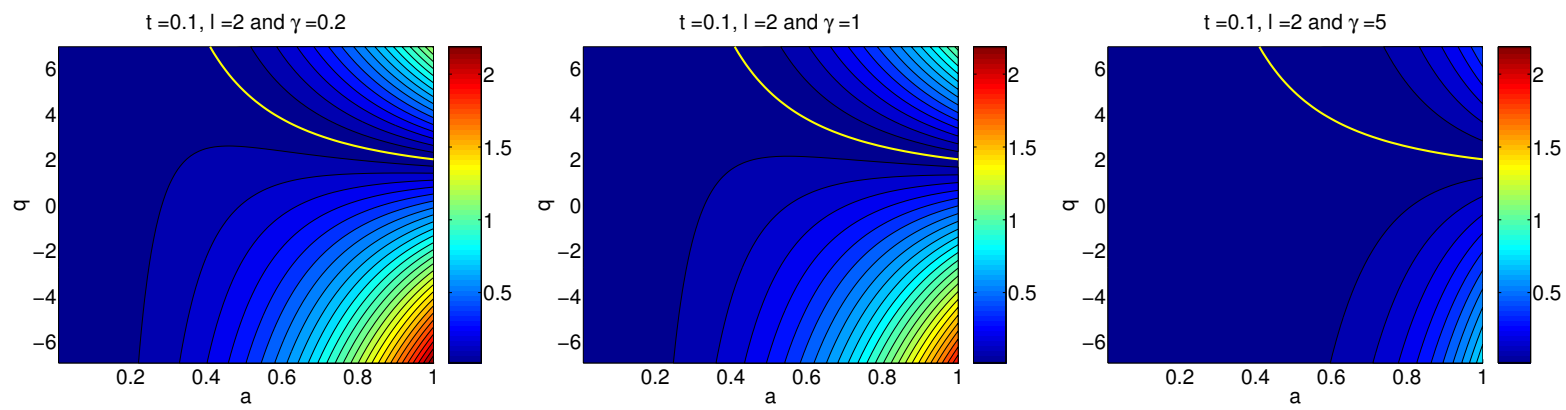

(b) $l=2$
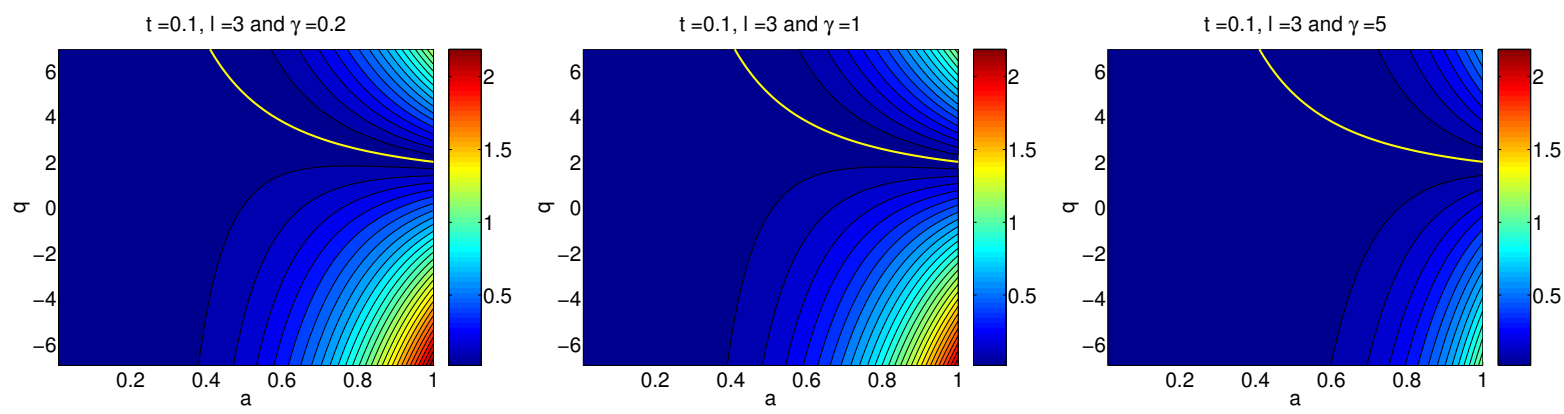

(c) $l=3$
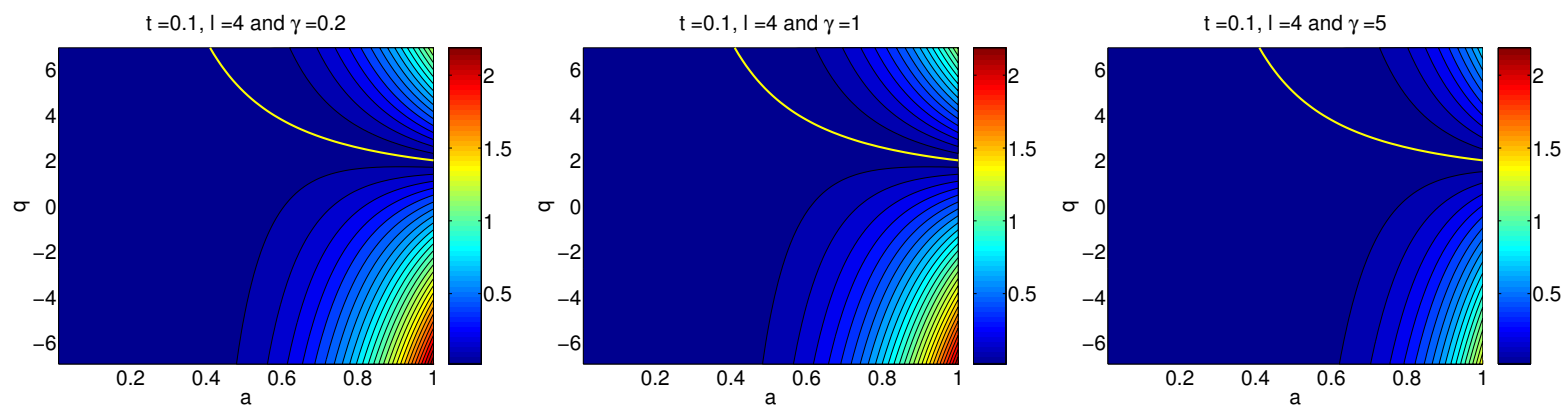

(d) $l=4$

Figure 2. Growth rates of the singular modes in the $\left(a, q_{0}\right)$ plane as a function of $\gamma=0.2,1,5$ and $l=1,2,3,4$ for $t=0.1$. 
The general calculation of $M$ for a 2x2 matrix $A$ is given in Appendix B of Rivière et al (2001): the resolvant is

$$
M=\frac{-1}{a_{+}-a_{-}}\left[\begin{array}{cc}
a_{-} e^{\sigma_{+} t}-a_{+} e^{\sigma_{-} t} & -e^{\sigma_{+} t}+e^{\sigma_{-} t} \\
a_{+} a_{-}\left(e^{\sigma_{+} t}-e^{\sigma_{-} t}\right) & -a_{+} e^{\sigma_{+} t}+a_{-} e^{\sigma_{-} t}
\end{array}\right]=\left[\begin{array}{cc}
c_{1} & c_{2} \\
d_{1} & d_{2}
\end{array}\right] .
$$

The eigenvalues $\mu_{1,2}$ of $M^{*} M$ are given in the Appendix of Fisher (1998):

$$
\mu_{1,2}=\frac{X_{11}+X_{22} \pm \sqrt{\Delta_{2}}}{2}
$$

with $X_{i j}=c_{i}^{*} c_{j}+d_{i}^{*} d_{j}$ and $\Delta_{2}=\left(X_{11}-X_{22}\right)^{2}+4 X_{12} X_{21}$. They are associated with the eigenvectors

$$
V_{1}=\left[\begin{array}{c}
1 \\
\frac{\sqrt{\Delta_{2}}-X_{11}+X_{22}}{2 X_{12}}
\end{array}\right] \text { and } V_{2}=\left[\begin{array}{c}
\frac{X_{11}-X_{22}-\sqrt{\Delta_{2}}}{2 X_{21}} \\
1
\end{array}\right]
$$

Figures 2 and 3 show the singular growth rates $\left(\frac{1}{2 t} \log (\lambda)\right)$ for $l=1,2,3,4$ and $\gamma=0.2,1,5$ in the $\left(a, q_{0}\right)$ plane at $t=0.1$ and 10 . The singular growth rates, which depend on time, are defined here as $\frac{1}{2 t} \log (\lambda)$ where $\lambda$ is the largest eigenvalue of $M^{*} M$. This allows a direct comparison with normal mode growth rates.

Firstly, for short times, one notices on figure 2 that singular modes are most unstable for narrow external annuli and intense cores with negative vorticity. In this case, the core circulation is much larger than that of the annulus which is very narrow. The maximal singular growth rates are then three times as large as the maximal growth rates of normal modes and specifically, the most unstable singular modes at short times lie in a region of normal mode stability. For short times, the asymptotic form of $M^{*} M$ is $I d+\left(A+A^{*}\right) t$ which has eigenvalues $\lambda_{a}=1+\left|A_{12}-A_{21}\right| t$ (see again Rivière et al (2001)). This asymptotic value provides an explanation for the maximum instability of singular modes at short times : since $\left|A_{12}-A_{21}\right|=\left[\frac{l}{a}-l a\left(q_{0}-1\right)\right] K_{l}(\gamma) I_{l}(\gamma a)$, the singular modes increase for $a \rightarrow 1$ when $l$ and $\left|q_{0}-2\right|$ increase. The zeros of this equation are $q_{0}=1+\frac{1}{a^{2}}$ which correspond to the solid yellow line in figure 2 and they represent a good approximation of the null growth rates of the singular modes.

Secondly for longer time $(t=100)$ not shown here, we note that the singular growth rates evolve towards the normal mode growth rates. This has been observed in many other studies. As Rivière et al (2001) have shown, the largest eigenvalue of $M^{*} M$ is

$$
\lambda_{+}=\frac{\left(1+\left|a_{+}\right|^{2}\right)\left(1+\left|a_{-}\right|^{2}\right)}{\left|a_{-}-a_{+}\right|^{2}} e^{2 \operatorname{Re}\left(\sigma_{+}\right) t},
$$

for $t>>1\left(\operatorname{Re}\left(\sigma_{+}\right)\right.$corresponds to the real part of $\left.\sigma_{+}\right)$. Here $\eta_{+}\left(1, a_{+}\right)$is the fastestgrowing normal mode, associated with the growth rate $\sigma_{+}$. At long time the singular eigenvalue $\lambda_{+}$is associated with the singular eigenvector $\eta_{\infty}\left(a_{+},-1\right)$ which is the biorthogonal of $\eta_{+}$. It is straightforward to show that $\frac{1}{2 t} \log (\lambda) \rightarrow \operatorname{Re}\left(\sigma_{+}\right)$when $t \rightarrow \infty$. 

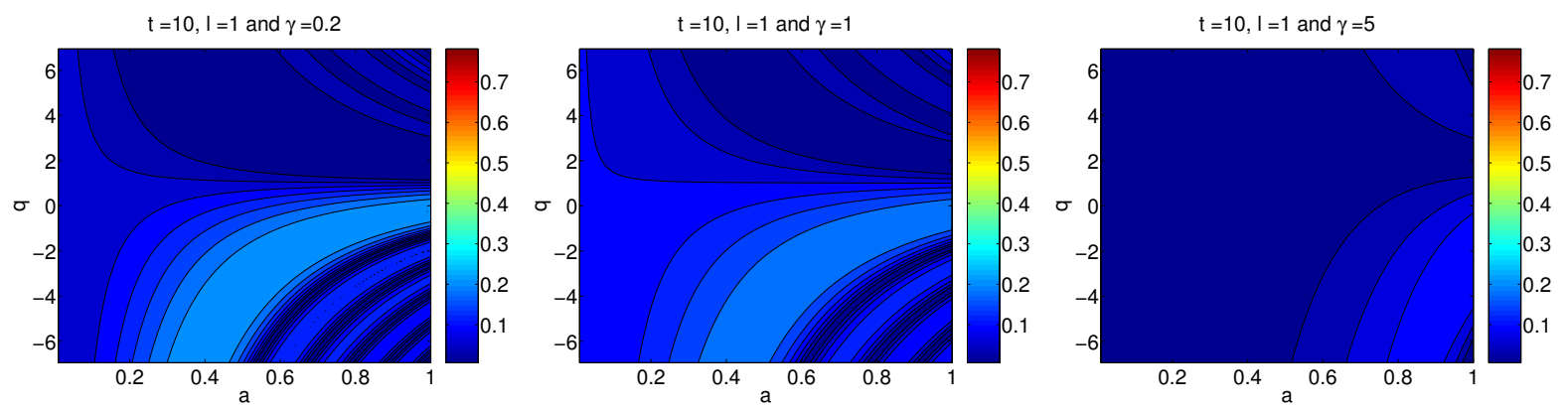

(a) $l=1$
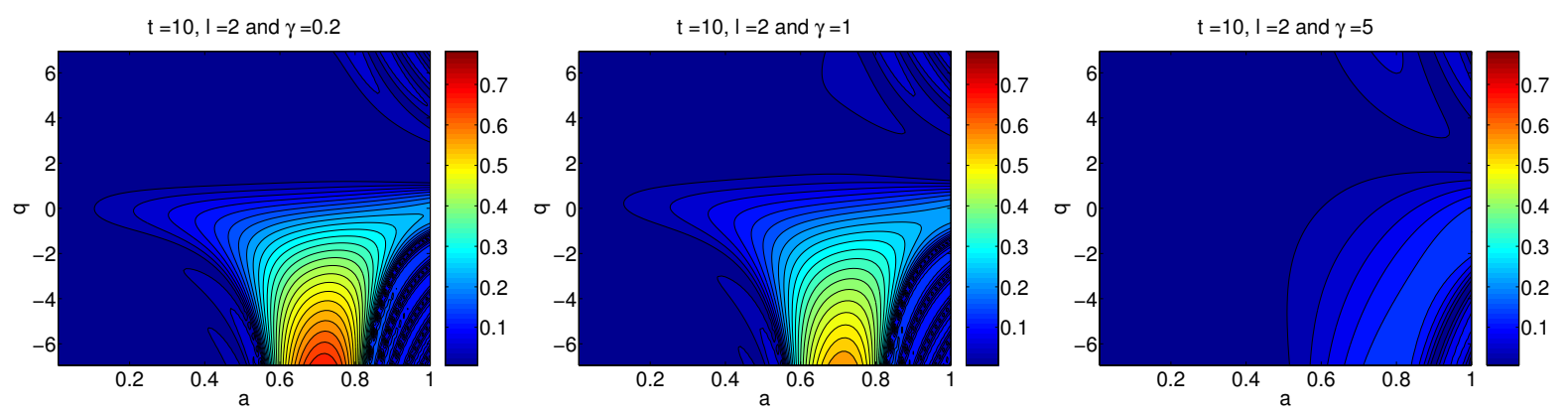

(b) $l=2$
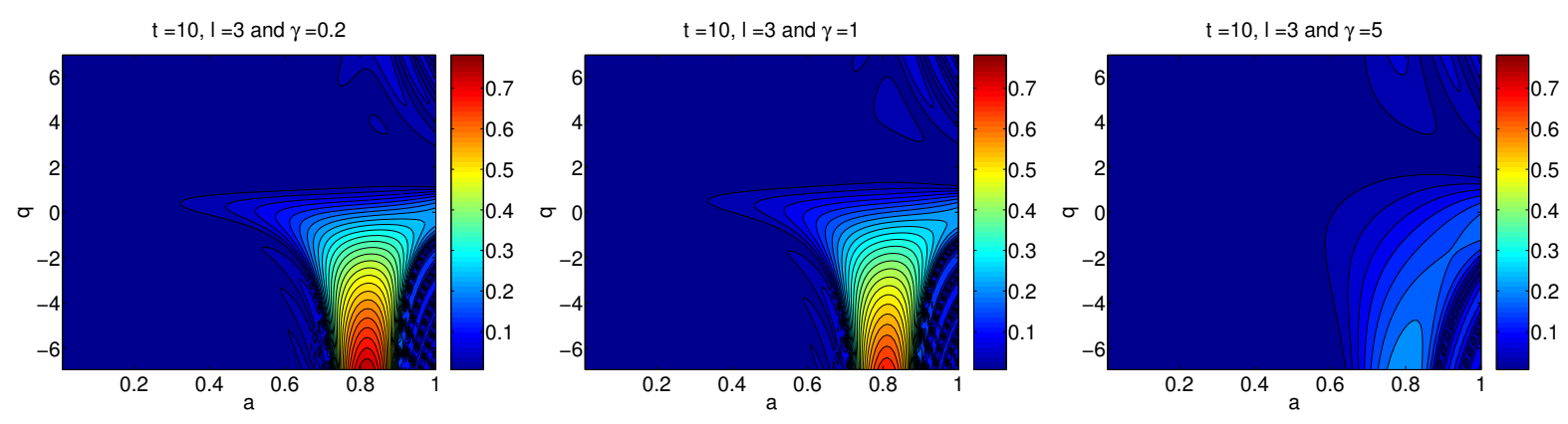

(c) $l=3$
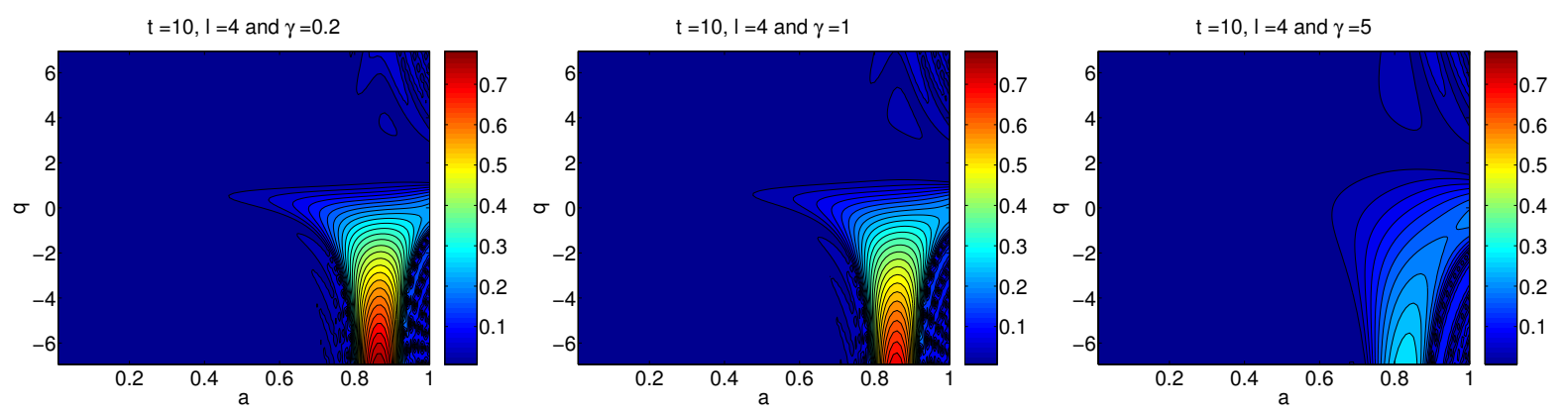

(d) $l=4$

Figure 3. Growth rates of the singular modes in the $\left(a, q_{0}\right)$ plane as a function of $\gamma=0.2,1,5$ and $l=1,2,3,4$ for $t=10$. 

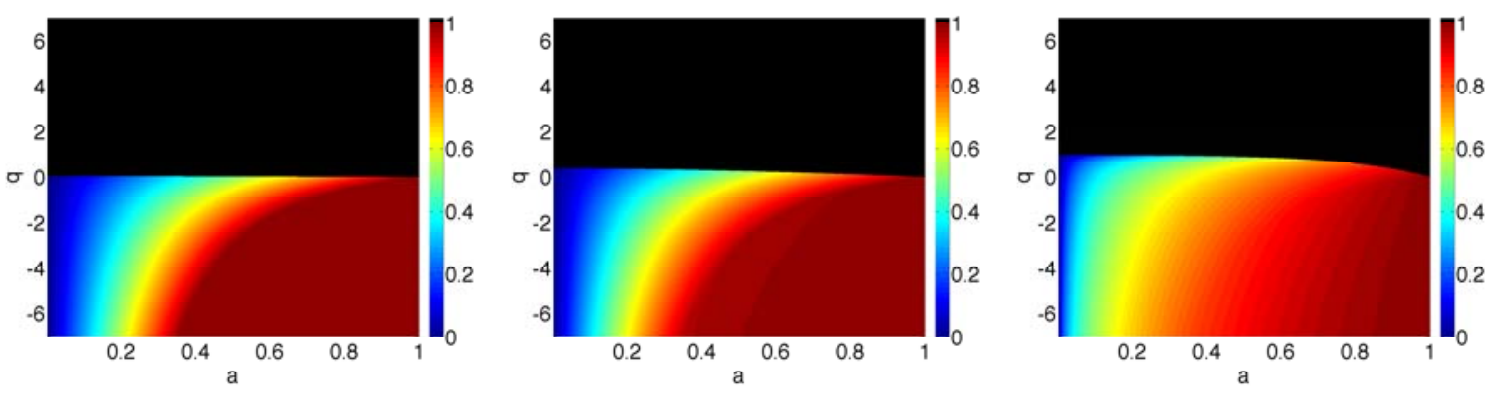

(a) $l=1$
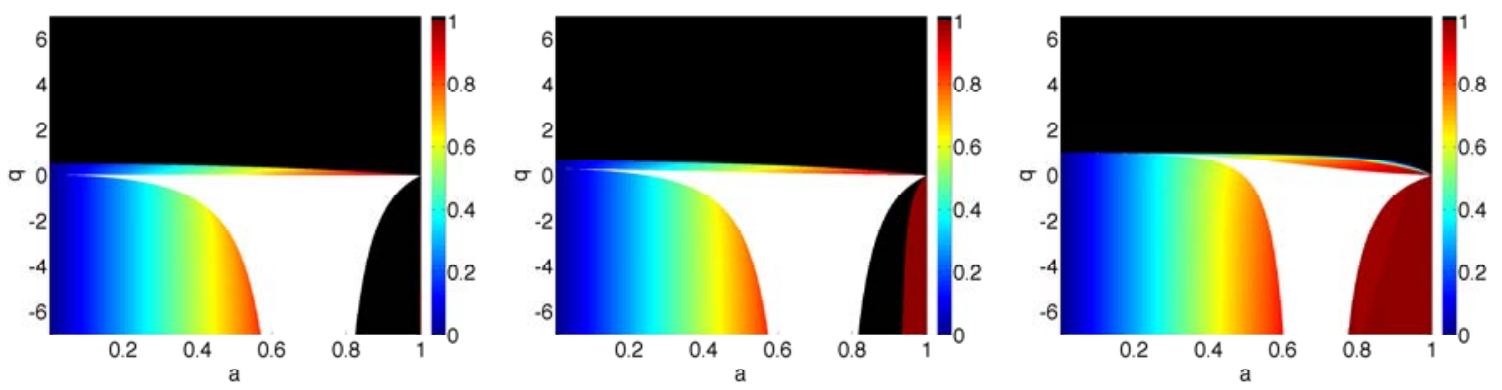

(b) $l=2$
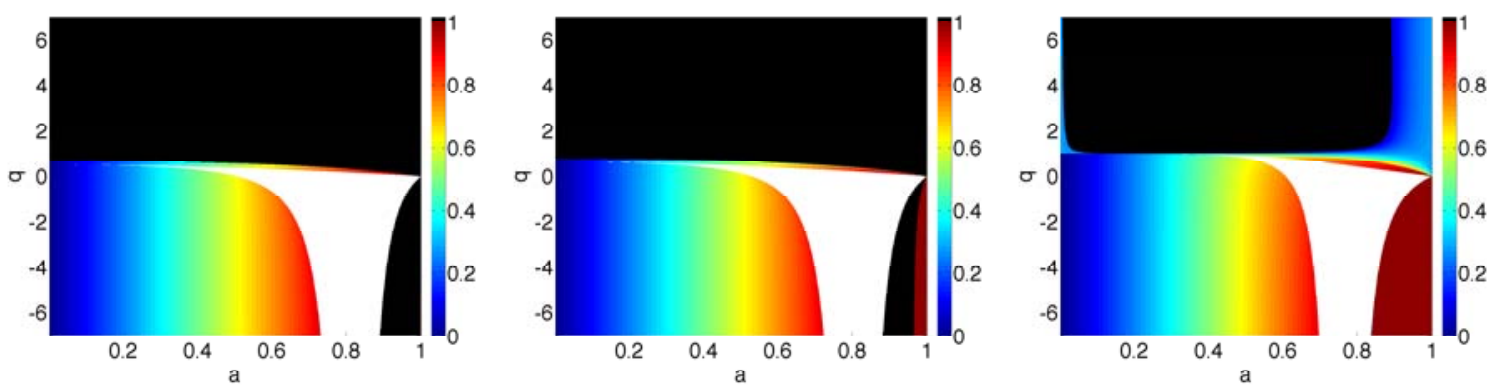

(c) $l=3$
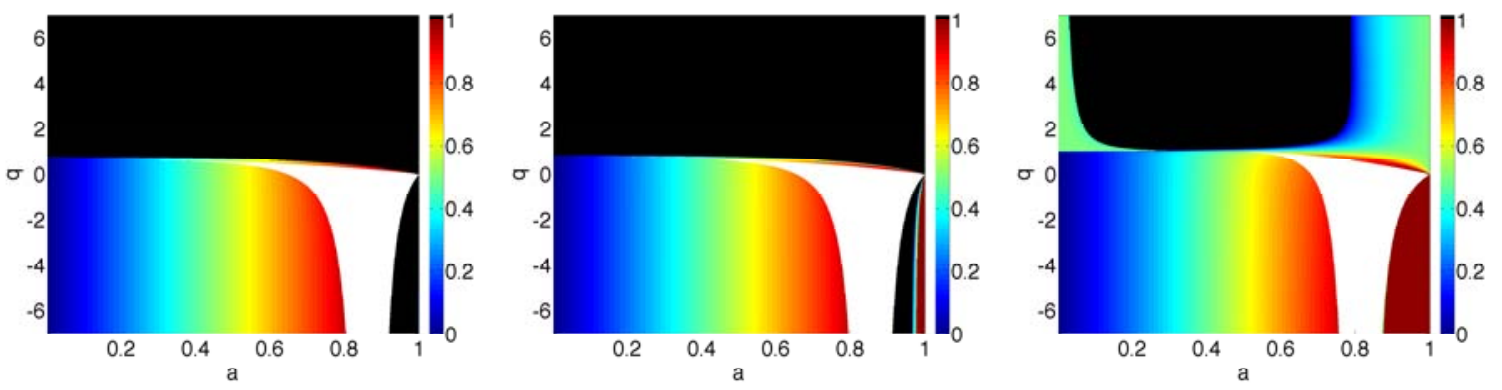

(d) $l=4$

Figure 4. Value of the critical radius in the $\left(a, q_{0}\right)$ plane for $\sigma_{+}$as a function of $\gamma=0.2,1,5$ and $l=1,2,3,4$ (black areas mean that the critical radius is outside the Rankine vortex, white areas correspond to the case of unstable normal mode). 

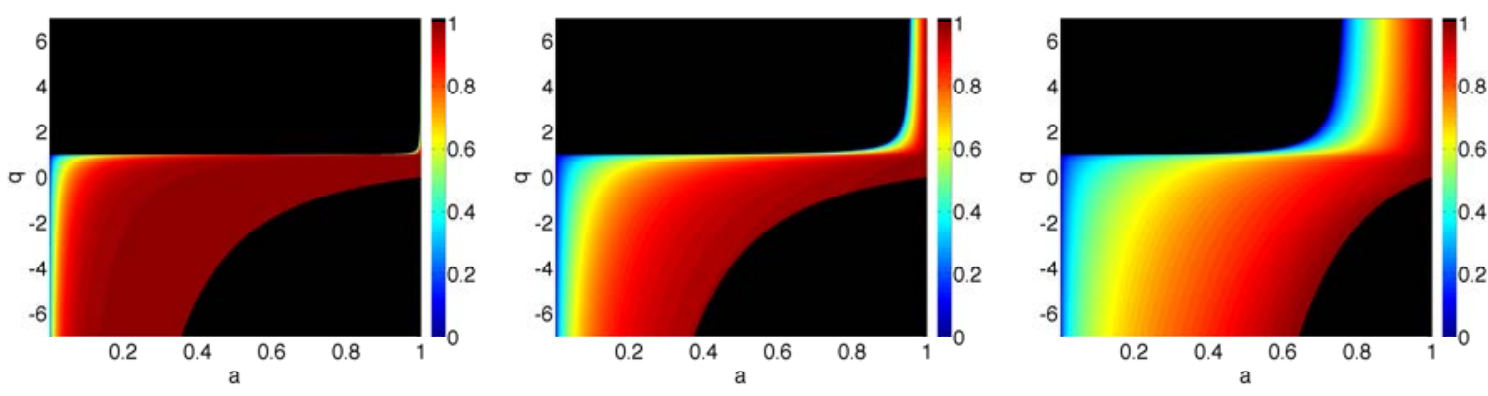

(a) $l=1$
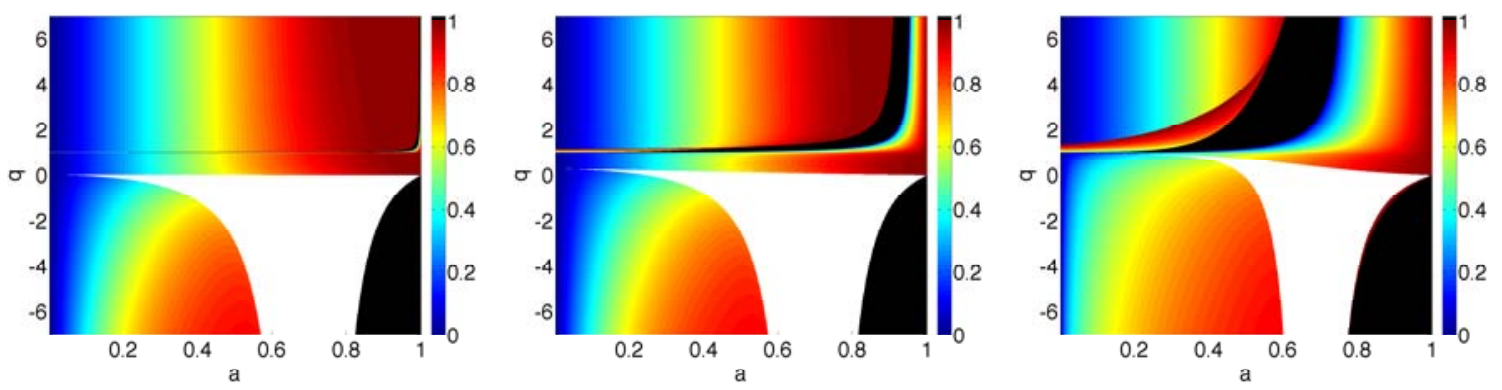

(b) $l=2$
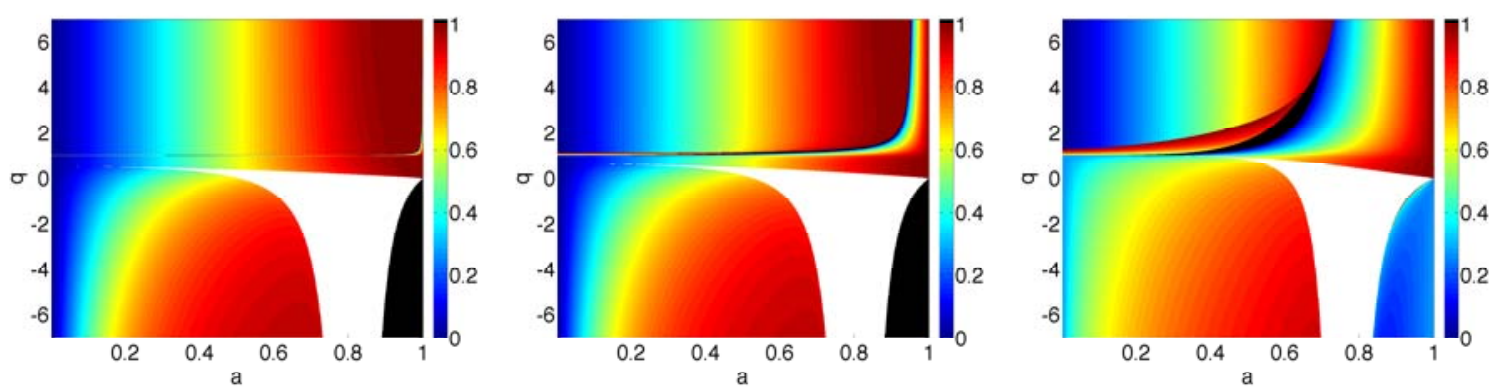

(c) $l=3$
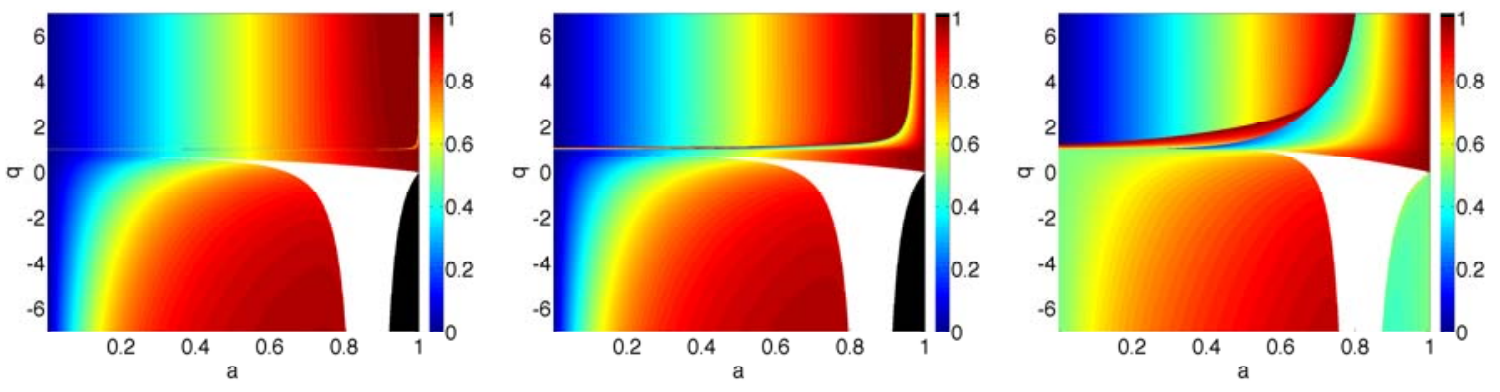

(d) $l=4$

Figure 5. Value of the critical radius in the $\left(a, q_{0}\right)$ plane for $\sigma_{-}$as a function of $\gamma=0.2,1,5$ and $l=1,2,3,4$ (black areas mean that the critical radius is outside the Rankine vortex, white areas correspond to the case of unstable normal mode). 


\section{The critical layer}

For vortices linearly stable with normal modes, perturbations can spiral (in vorticity) around the critical layer. Balmforth et al (2001) have shown that vortices can become unstable near this critical layer if a forcing is applied. This is of interest for our future study of parametric instability. This critical layer is defined by the radius at which the rotation frequency of the waves matches the angular velocity of the fluid. In our, case the critical radius is defined as:

$$
\frac{\bar{U}_{\theta}\left(r_{c}\right)}{r_{c}}=-\frac{\sigma}{i l} \text {. }
$$

We numerically calculate the value of the first critical radius (if it exists) for each stable normal modes (pure imaginary growth rate), by first critical radius we mean the smallest radius if it exist more than one. Calculations show that there is always a critical layer which could be inside or outside the Rankine. For $q_{0}>1$ the first critical layer is mainly outside or inside the Rankine vortex depending on the normal mode (see figures 4 and 5). On the contrary if $q_{0}<1$ it exists a critical radius inside the Rankine for almost every case. In addition the existence of the critical layer inside the annulus is favored by a large value of $\gamma$ wich is consistent with the impact of $\gamma$ on the increase of the range of $\bar{U}_{\theta} / r$ inside the Rankine and as a results the ability of having a critical radius inside the Rankine. Finally for the mode 2, 3 and 4 at the boundary of the instability region we have a convergence for the value of the critical radius for the two normal modes which is consistent with the equality of these modes at the boundary.

Figure 6 presents the evolution of a potential vorticity perturbation in the annulus performed with a spectral QG model. The parameters chosen allow the existence of a critical layer inside the annulus. We examine the consequence of this on the plot of potential vorticity anomaly; PVA lines wrap around as spiral lines, as shown in a previous paper (Bernoff and Lingevitch 1994).

\section{Conclusion}

This study has shown that in a QG one-and-a-half layer model, singular modes can grow on circular two-step Rankine vortices, via barotropic instability, as they do on two-layer Rankine vortices via baroclinic instability. The same conclusions apply, that is, short wave instability dominates at small times, with large growth rates, and the singular growth rates converge towards those of exponential modes at long times. It has also been shown that critical layers play an essential role in the time evolution of potential vorticity of the perturbation, when the vortex is linearly stable to normal modes. This role is that described in other studies, i.e. the wrapping of perturbation potential vorticity around spiral lines. 

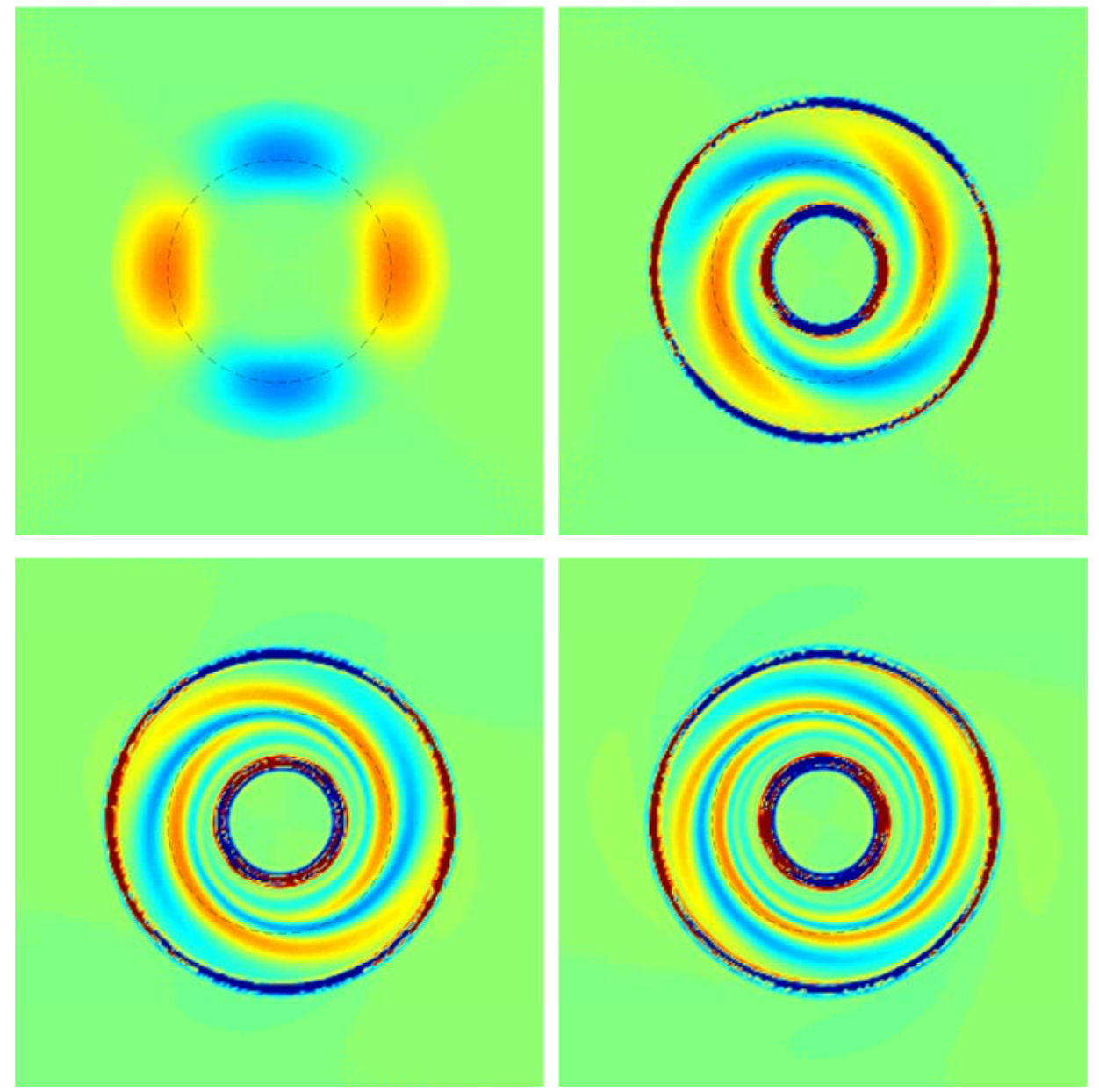

Figure 6. Evolution of a mode 2 potential vorticity anomaly in the annulus, with $q_{0}=-2, a=0.35$ and $r_{c}=0.66$ (the dashed line corresponds to the position of the critical layer).

This study could be extended to compute the parametric instability of this vortex when its mean flow varies sinusoidally with time (Carton et al 2010b). In that respect, we also wish to determine the role of the critical layer as the flow becomes forced (in relation with the work by Balmforth et al (2001)). We alos wish to extend the study of the case where more than one critical layer could exist and to compare it to the case of a single stair Rankine. We also plan to extend this study to the case where the mean flow varies stochastically with time.

\section{Acknowledgments}

We gratefully thank the reviewers for their helpful comments. This study was achieved in partial fulfillment of X Perrot's PhD thesis founded by a MNESR grant. 


\section{References}

Balmforth N J, Llewellyn Smith S G and Young W R 2001 Disturbing vortices J. Fluid Mech. 426 95-133

Bernoff A J and Lingevitch J F 1994 Rapid relaxation of an axisymmetric vortex Phys. Fluids 6 $3717-3723$

Carton X 1992 On the merger of shielded vortices Europhys. Lett. 18 697-703

Carton X and Corréard S 1999 IUTAM Symp. on Simulation and Identification of Organized Structures in Flows (Kluwer Academic Publishers) p 181

Carton X, Flierl G R, Perrot X, Meunier T and Sokolovskiy M A 2010a Explosive instability of geostrophic vortices. part 1. baroclinic instability Theor. Comp. Fluid Dyn. 24 125-130

Carton X, Meunier T, Flierl G R, Perrot X and Sokolovskiy M A 2010b Explosive instability of geostrophic vortices. part 2. parametric instability Theor. Comp. Fluid Dyn. 24 131-135

Charney J G and Stern M E 1962 On the stability of internal baroclinic jets in a rotating atmosphere J. Atmos. Sci. 19 159-172

Fischer C 1998 Linear amplification and error growth in the 2d eady problem with uniform potential vorticity J. Atmos. Sci. 55 3363-3380

Flierl G R 1988 On the instability of geostrophic vortices J. Fluid Mech. 197 349-388

Gent P R and McWilliams J C 1986 The instability of barotropic circular vortices Geophys. Astrophys. Fluid Dyn. 35 209-233

Morel Y G and Carton X 1994 Multipolar vortices in two-dimensional incompressible flows J. Fluid Mech. $26723-51$

Pedlosky J 1985 The instability of continuous heton clouds J. Atmos. Sci. 42 1477-1486

Rayleigh L 1880 On the stability, or instability, of certain fluid motions Proc. Roy. Soc. London 11 $57-70$

Rivière G, Hua B L and Klein P 2001 Influence of the $\beta$-effect on non-modal baroclinic instability Quart. J. Roy. Meteorol. Soc. 127 1375-1388

Sokolovskiy M A 1997 Stability of an axisymmetric three-layer vortex Izv. Atmos. Ocean Phys. 33 $16-26$

Stern M E 1987 Horizontal entrainment and detrainment in large-scale eddies J. Phys. Oceanogr. 17 1688-1695 